\title{
A loop theorem/Dehn's lemma for some orbifolds
}

\author{
JOSH BARNARD
}

\begin{abstract}
The equivariant loop theorem implies the existence of a loop theorem/Dehn's lemma for 3-orbifolds that are good (covered by a 3-manifold). In this note we prove a loop theorem/Dehn's lemma for any locally orientable 3-orbifold (good or bad) whose singular set is labeled with powers of 2 . The proof is modeled on the standard tower construction.
\end{abstract}

$57 \mathrm{M} 35$

We prove a version of the loop theorem and Dehn's lemma for a certain class of 3orbifolds, namely those which are locally orientable and have singular set labeled with powers of 2. As part of a program to extend Waldhausen's theorems on 3-manifolds to 3-orbifolds, Takeuchi and Yokoyama [4, Corollary 6.4] have proven a loop theorem for good 3-orbifolds (ie, those covered by a manifold) using the equivariant loop theorem for 3-manifolds, along with a generalization of normal surface theory. Thus the novelty of the proof given here is two-fold: it extends the loop theorem to certain bad 3-orbifolds, and it uses the more direct techniques of cutting and pasting, in the spirit of Papakyriakopoulos' original tower proof [3]. Our presentation and notation are modeled on those of Hatcher [2]. As a corollary we show that in a covering of an orbifold to which the loop theorem applies, orbifold incompressible 2-suborbifolds lift to orbifold incompressible 2-suborbifolds.

As should be expected, the assumption that the singular set is labeled with powers of two is directly related to the use of double covers in the standard tower proof. In particular, it is not at all clear that any modification of this proof would work for 3 -orbifolds with more general singular labels. This seems to be the main obstacle for settling the outstanding question of whether a loop theorem holds for 3-orbifolds in general. On the other hand, it should be possible, with some additional effort, to drop the assumption of local-orientability, as any non-locally orientable 3-orbifold is a quotient of a locally orientable one under an involution, and involutions enjoy the distinct advantage of having order two.

We begin by reviewing some basic notions about orbifolds, mostly to fix notation. We also prove some technical results, all of which are straight-forward generalizations of concepts common in 3-manifold theory. We henceforth let $Q$ denote a locally 
orientable 3-orbifold. We denote by $|Q|$ the underlying space of an orbifold $Q$, which will be a manifold because $Q$ is locally orientable. Also because $Q$ is locally orientable, the orbifold boundary $\partial Q$ of $Q$ coincides with the boundary of the underlying manifold, and we use the same notation for both. The singular set of $Q$ is denoted $\Sigma_{Q}$, or just $\Sigma$, depending on the context. We let $D(n)$ denote the quotient of the disc $D=\{z \in \mathbf{C} \mid$ $|z| \leq 1\}$ by the rotation $z \mapsto z^{n}$. We will work in the simplicial category, where we always assume that the singular set $\Sigma_{Q}$ is a subcomplex of $Q$. We refer the reader to Cooper, Hodgson and Kerckhoff [1] for the definitions of orbifold and orbifold fundamental group, which we denote by $\pi_{1}^{\text {orb }}(Q)$.

Definitions In a simplicial 3-orbifold $Q$, we define the orbifold regular neighborhood of a subcomplex $A$ to be a subcomplex $N(A)$ of $Q$ that strong deformation retracts onto $(N(A) \cap \Sigma) \cup A$. As usual, the simplices intersecting $A$ in the second barycentric subdivision provide the prototype for an orbifold regular neighborhood of $A$.

An orbifold embedding of an orbifold $X$ into $Q$ is a label-preserving map $f:\left(X, \Sigma_{X}\right) \rightarrow$ $\left(Q, \Sigma_{Q}\right)$ so that the associated map $|X| \rightarrow|Q|$ is an embedding transverse to $\Sigma_{Q}$. An orbifold embedding is proper if $f^{-1}(\partial Q)=\partial X$. A two-sided 2-suborbifold $F$ in a locally orientable 3-orbifold $Q$ is orbifold compressible if there is an orbifold embedded disc $(D(n), \partial D(n)) \rightarrow(Q, F)$ with $[\partial D(n)]^{n}$ nontrivial in $\pi_{1}^{\text {orb }}(F)$. Otherwise, $F$ is orbifold incompressible. Such a disc is an orbifold compressing disc.

In the case $F \neq \mathbf{R} P^{2}$ and $Q$ is good, saying $[\partial D(n)]^{n}$ nontrivial in $\pi_{1}^{\text {orb }}(F)$ is equivalent to saying that $\partial D(n)$ does not bound an orbifold disc (with singular point labeled $n$ ) in $F$.

A loop $\gamma: S^{1} \rightarrow Q \backslash \Sigma$ is orbifold null-homotopic if it lifts to the universal orbifold cover $\widetilde{Q}$. A map $f: D \rightarrow Q$ is a wound disc if $f(D) \cap \Sigma$ is a finite set of points $x_{1}, \ldots, x_{k}$ in the interiors of edges of $\Sigma$ labeled $n_{1}, \ldots, n_{k}$, respectively, at which $f$ is transverse to $\Sigma$ and such that, for each $i, f^{-1}\left(x_{i}\right)$ is a single point $p_{i}$ contained in an open neighborhood $U_{i} \subset D$ on which $f$ acts as the map $z \mapsto z^{n_{i}}$. In other words, for each $x_{i} \in f(D) \cap \Sigma$, there exist homeomorphisms $\left(U_{i}, p_{i}\right) \cong(D, 0)$ and $\left(f\left(U_{i}\right), x_{i}\right) \cong(D, 0)$ such that the following diagram commutes:

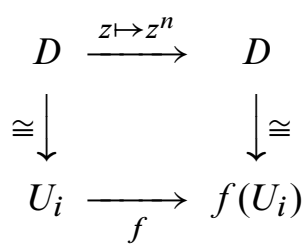

An orbifold null-homotopy of a loop $\gamma$ is a wound disc with $\left.f\right|_{\partial D}=\gamma$. 
Proposition A loop $\gamma$ is orbifold null-homotopic in $Q$ if and only if there is an orbifold null-homotopy of $\gamma$ in $Q$.

Proof Suppose $\gamma$ is orbifold null-homotopic in $Q$, let $\widetilde{Q}$ be the universal orbifold cover of $Q$, and let $\tilde{\gamma}$ be a lift of $\gamma$. If we can find a wound disc $f: D \rightarrow \widetilde{Q}$, then we can homotope $f$ so that each winding point of $f(D)$ has a neighborhood equivariant under the local group actions corresponding to the covering $p: \widetilde{Q} \rightarrow Q$. It follows that the property of being wound is preserved under composition with orbifold covering projections, so to prove that $\gamma$ is part of a null-homotopy, it suffices to find a wound disc in $\widetilde{Q}$ whose boundary projects to $\gamma$.

If $\widetilde{Q}$ has nonempty singular set $\Sigma \widetilde{Q}$, then the fundamental group of $\widetilde{Q} \backslash \Sigma \widetilde{Q}$ is normally generated by paths $\alpha_{i}=\delta_{i}^{-1} \lambda_{i} \delta_{i}$, where $\lambda_{i}$ is a small loop running once around a singular arc labeled $n_{i}$, and $\delta_{i}$ is a path running from the basepoint to $\lambda_{i}$. Adding in the relations $\alpha_{i}^{n_{i}}=1$ makes $\tilde{\gamma}$ trivial. It follows that the class represented by $\tilde{\gamma}$ is contained in the normal subgroup of $\pi_{1}(\widetilde{Q} \backslash \Sigma \widetilde{Q})$ generated by the terms $\alpha_{i}^{n_{i}}$, so we may represent $[\tilde{\gamma}]$ as a product of the form $w_{0} A_{i_{1}} w_{1} A_{i_{2}} \cdots A_{i_{t}} w_{t}$, where $A_{i}=\delta_{i}^{-1} \lambda_{i}^{n_{i}} \delta_{i}$ and $w_{0} w_{1} \cdots w_{t}$ is trivial in $\pi_{1}(\widetilde{Q} \backslash \Sigma \widetilde{Q})$.

Note that a map of a circle onto a path representing $A_{i}$ extends over a disc, by first "collapsing" the parts of the circle mapping to $\delta_{i}^{ \pm 1}$, and then extending over the remaining disc by the map $z \mapsto z^{n_{i}}$. Doing this for each $A_{i}$ leaves a loop representing $w_{0} w_{1} \cdots w_{t}$ and thus bounding a disc in $\widetilde{Q} \backslash \Sigma \widetilde{Q}$. There is therefore a wound disc in $\widetilde{Q}$ with boundary $\tilde{\gamma}$.

Conversely, suppose that we have a wound disc $f: D \rightarrow Q$ with $f(D) \cap \Sigma=$ $\left\{x_{1}, \ldots, x_{k}\right\}$. If $\sigma_{i}$ is a loop about $f^{-1}\left(x_{i}\right)$, then $f\left(\sigma_{i}\right)$ is a loop about $x_{i}$ traversed $n_{i}$ times. Such loops lift to $\widetilde{Q}$. Since these $\sigma_{i}$ generate $\pi_{1}\left(D \backslash f^{-1}(\Sigma)\right)$, it follows that $\left.f\right|_{\partial D}$ is null-homotopic in $Q$.

Homotopy Lifting Lemma Given an orbifold null-homotopy $f: D \rightarrow Q$ of a loop $\gamma$, an orbifold covering $p: \hat{Q} \rightarrow Q$, and a lift $\hat{\gamma} \subset \widehat{Q}$ of $\gamma$, there is a map $\hat{f}: D \rightarrow \widehat{Q}$ such that $p \circ \hat{f}=f$ and $\hat{f}(\partial D)=\hat{\gamma}$.

Proof Let $f, \gamma, p$, and $\hat{\gamma}$ be as in the statement, and let $x_{1}, \ldots, x_{k}$ and $n_{1}, \ldots, n_{k}$ be points of $f(D) \cap \Sigma$ with corresponding labels $n_{1}, \ldots, n_{k}$. That $f$ lifts to a map $\widehat{f}: D \backslash\left\{x_{1}, \ldots, x_{k}\right\} \rightarrow \widehat{Q} \backslash \widehat{\Sigma}$ follows as in the last paragraph of the proof above.

Now consider a point $f\left(x_{i}\right) \in \Sigma$. We need only extend the lift $\hat{f}$ continuously to $x_{i}$. To this end, let $B^{3}=\left\{(z, x) \in \mathbf{C} \times\left.\mathbf{R}|| z\right|^{2}+x^{2}<1\right\}$, and note that because $\widehat{Q}$ is an orbifold cover of $Q$, there is a $B^{3}$-neighborhood $V_{i}$ of $f\left(x_{i}\right)$ in $Q$ that is covered 
by a $B^{3}$-neighborhood $\widehat{V}_{i}$ in $\widehat{Q}$ on which $p$ acts as the map $(z, x) \mapsto\left(z^{d_{i}}, x\right)$ for some $d_{i}$ dividing $n_{i}$. Then on a neighborhood $U_{i}$ of $x_{i}$ we have the following:

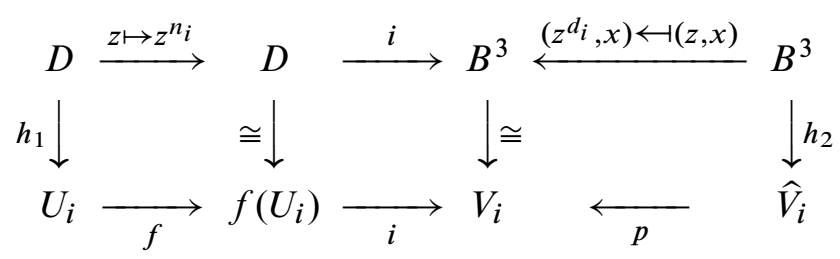

where all of the vertical maps are homeomorphisms. The map $g(z)=\left(z^{n_{i} / d_{i}}, 0\right)$ along the top row is well-defined, since $d_{i} \mid n_{i}$. We may thus define $\hat{f}: U_{i} \rightarrow \widehat{V}_{i}$ to be $\hat{f}=h_{2} \circ g \circ h_{1}^{-1}$. Moreover, this lift agrees (up to isotopy) with the previously defined $\widehat{f}$, which is unique (up to isotopy). We may therefore extend $\hat{f}$ over all of $D$.

Theorem Let $Q$ be a locally orientable 3-orbifold with all singular arcs labeled with powers of 2. Suppose $K=\operatorname{Ker}\left\{i_{*}: \pi_{1}^{\mathrm{orb}}(\partial Q) \rightarrow \pi_{1}^{\mathrm{orb}}(Q)\right\}$ is nontrivial, and let $N \subsetneq K$ be a proper normal subgroup of $K$. Then there is a properly embedded orbifold disc $D(n)$ in $Q$ with the property that $[\partial D(n)]^{n} \notin N$.

Proof Since $N$ is proper in $K$, there is a null-homotopy $f:(D, \partial D) \rightarrow(Q, \partial Q)$ with $[f(\partial D)] \notin N \subset \pi_{1}^{\text {orb }}(\partial Q)$. We may triangulate $Q$ and $D$ so that the singular set $\Sigma$ of $Q$ is a subcomplex and $f$ is homotopic to a simplicial map $f_{0}$.

Let $N_{0}$ be an orbifold regular neighborhood of the image $J_{0}$ of $f_{0}$. Suppose $N_{0}$ has a (connected) two-fold orbifold cover $p_{1}: Q_{1} \rightarrow N_{0}$. Note that $p_{1}^{-1}\left(J_{0}\right)$ is connected, since $N_{0}$ retracts onto $J_{0}$, and we can lift this retraction to one of $Q_{1}$ onto $p_{1}^{-1}\left(J_{0}\right)$.

By the homotopy lifting lemma, $f_{0}$ lifts to a map $f_{1}: D \rightarrow Q_{1}$ whose image $J_{1}$ has orbifold regular neighborhood $N_{1}$. Continue, as long as possible, to take orbifold regular neighborhoods and orbifold double covers. We will show that this process terminates, so that for some $k, N_{k}$ has no orbifold double cover.

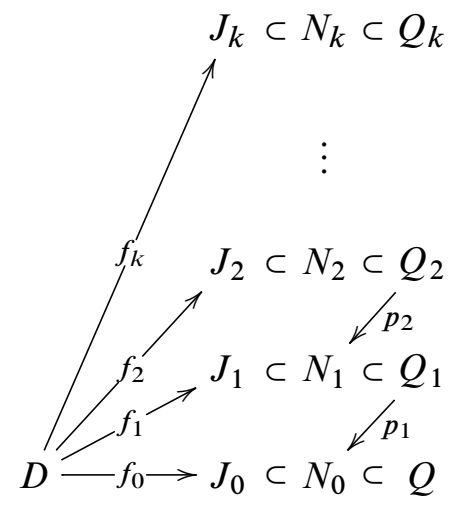


To this end, consider a typical orbifold double cover $p_{i}: Q_{i} \rightarrow N_{i-1}$, and let $\rho$ be the corresponding orbifold covering transformation. Then $J_{i} \cap \rho\left(J_{i}\right) \neq \varnothing$, since $J_{i} \cup \rho\left(J_{i}\right)=p_{i}^{-1}\left(J_{i-1}\right)$ is connected. (Note that we may have $J_{i}=\rho\left(J_{i}\right)$.) If $\rho$ has no fixed points in $J_{i}$, then there are two simplices in the intersection with disjoint interiors that are interchanged by $\rho$. In passing to $J_{i-1}$, these simplices are identified. Hence, $J_{i}$ has strictly more simplices than $J_{i-1}$.

On the other hand, suppose $\rho$ has a fixed point $x \in J_{i}$. Then in a 3-ball neighborhood of $x, \rho$ acts as rotation by $\pi$ about an axis passing through $x$ and transverse to both $J_{i}$ and $\rho\left(J_{i}\right)$. It follows that $J_{i} \cap \rho\left(J_{i}\right)$ contains (at least) two edges sharing the vertex $x$. These are interchanged by $\rho$, from which we deduce, as before, that $J_{i}$ has strictly more simplices than $J_{i-1}$. The claim follows from the fact that the number of simplices in any $J_{i}$ is bounded above by the number of simplices in the triangulation on $D$.

We have now an orbifold $N_{k}$ that is a regular neighborhood of a mapped-in disc and that has no two-fold orbifold covers. Note that this implies that the underlying manifold has no two-fold covers, so $\operatorname{Hom}\left(H_{1}\left|N_{k}\right|, \mathbf{Z}_{2}\right)=\{0\}$. Using Poincaré-Lefschetz duality, universal coefficients, and dual vector spaces, we deduce that in the following piece of a long exact sequence

$$
H_{2}\left(\left|N_{k}\right|,\left|\partial N_{k}\right| ; \mathbf{Z}_{2}\right) \rightarrow H_{1}\left(\left|\partial N_{k}\right| ; \mathbf{Z}_{2}\right) \rightarrow H_{1}\left(\left|N_{k}\right| ; \mathbf{Z}_{2}\right),
$$

the outermost terms are both zero. Thus $H_{1}\left(\left|\partial N_{k}\right| ; \mathbf{Z}_{2}\right)=\{0\}$, which implies that $N_{k}$ has boundary components that are topological spheres.

Let $A$ be the component of $\partial N_{k}$ on which $f_{k}(\partial D)$ lies, and let $A^{\prime}$ be the preimage $\left(p_{1} \circ \cdots \circ p_{k}\right)^{-1}(\partial Q) \subset A$. Note that $A^{\prime}$ contains no singular points, since the projection of $A^{\prime}$ into $\partial Q$ is an orbifold regular neighborhood of a closed loop in $\partial Q$ avoiding singular points. Thus $A^{\prime}$ is a nonsingular planar surface with (orbifold) fundamental group generated by simple closed curves. Now $f_{k}(\partial D)$ is a word in these generators with the property that its projection into $\pi_{1}^{\text {orb }}(\partial Q)$ is not contained in $N$. It follows that some generator also has this property.

Unlike the manifold case, it is not immediately clear that such a curve bounds an embedded orbifold disc in $N_{k}$ (there may be more than one singular arc passing through each of the two disc components of $A$ bounded by the curve). It is not even obvious that we may choose this simple closed curve to represent a trivial element in $\pi_{1}^{\text {orb }}\left(N_{k}\right)$, let alone to have its projection contained in $K$. In order to get such an embedded orbifold disc, we use the hypothesis on the labels of the singular set of $Q$.

Let $\Gamma$ be a graph containing one vertex for each boundary component of $N_{k}$, and with the property that two vertices are connected by one edge for each singular arc in $N_{k}$ joining the two corresponding boundary components. Suppose (for contradiction) this 

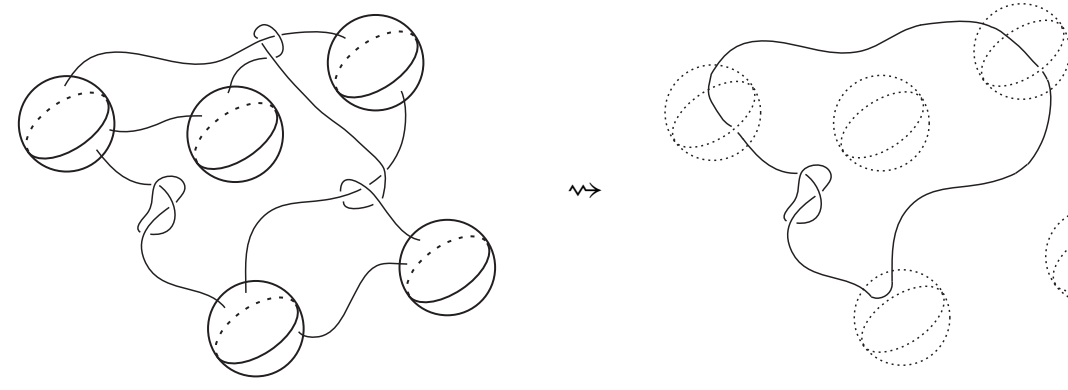

Figure 1: Obtaining a single loop labeled 2

graph contains a circuit, and choose one with a minimal number of vertices. Then we construct an orbifold $\mathcal{O}$ from $N_{k}$ by changing edge labels and capping boundary components as follows: Label with a 2 every singular arc in $N_{k}$ corresponding to an edge in the circuit, make all other arcs nonsingular, and cap off all boundary components with balls. In the balls attached to those boundary components corresponding to vertices in the circuit, insert a single arc labeled 2 joining the two singular arcs intersecting the boundary of the ball (there are only two arcs intersecting the boundary by the minimality assumption above). Then $\mathcal{O}$ is a closed orbifold containing a single (possibly knotted) loop $\ell$ labeled 2; see Figure 1.

Note that $|\mathcal{O}|$ is obtained from $\left|N_{k}\right|$ by capping off sphere boundary components with balls. This has no effect on first homology, so it follows that $H_{1}\left(|\mathcal{O}| ; \mathbf{Z}_{2}\right)=$ $H_{1}\left(\left|N_{k}\right| ; \mathbf{Z}_{2}\right)=0$. In particular, $\ell$ is null-homologous (with $\mathbf{Z}_{2}$ coefficients) so there is a well-defined map $h: \pi_{1}(|\mathcal{O}| \backslash \ell) \rightarrow \mathbf{Z}_{2}$ where $h([\gamma])$ is the mod 2 linking number of $\gamma$ with $\ell$. This implies that $|\mathcal{O}| \backslash \ell$ has a two-fold cover that unwraps around $\ell$. Since $\ell$ is labeled 2, this cover induces a two-fold orbifold cover $\widetilde{\mathcal{O}}$ of $\mathcal{O}$.

Now since the balls glued onto the boundary components of $N_{k}$ lift to balls in the cover, we may remove them from $\mathcal{O}$ while preserving the orbifold cover on the remaining orbifold. Once this is done, we observe that the orbifold covering projection is locally a homeomorphism away from the lift of $\ell$. We may therefore restore the labels on the singular set not in $\ell$, placing the same labels on the lifts, and still have an orbifold cover. Finally, since each component of $\Sigma$ giving rise to a part of $\ell$ has label divisible by two, we may restore the labels on these components, giving the lifted components half the label of their projections. We thus obtain a two-fold orbifold cover of $N_{k}$, contrary to assumption, and deduce that the singular set of $N_{k}$ contains no circuits.

It follows from this that any simple closed curve $\gamma$ in $A$ bounds an embedded nonsingular disc in $N_{k}$; see Figure 2. 


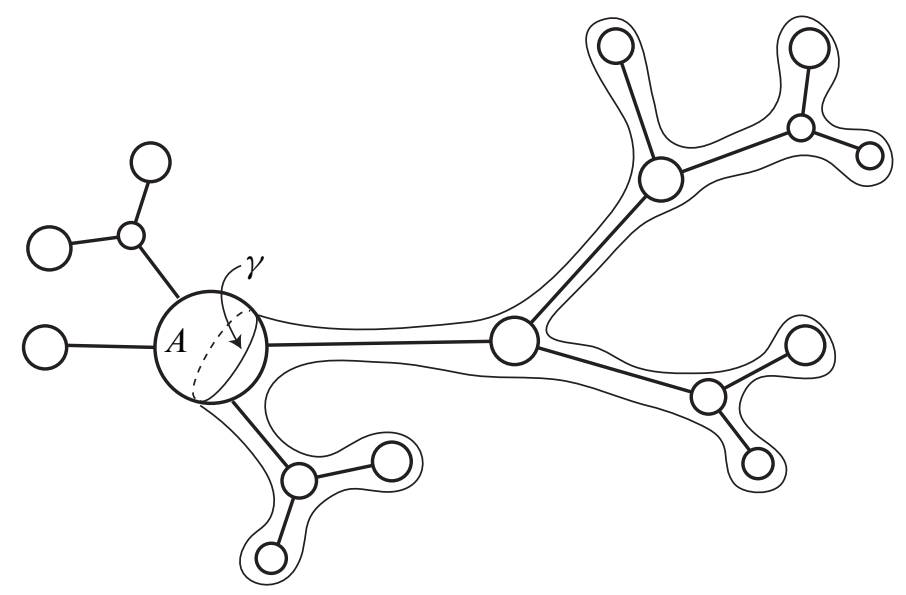

Figure 2: An embedded disc with boundary $\gamma$

We now show that we may obtain orbifold compressing discs in each $N_{i}$ by pushing this disc down the tower. In fact we will show that the disc obtained at each step will contain at most one singular point. Assume we have a properly embedded orbifold compressing disc $S$ in $N_{i}$ with a singular point labeled $n$. Suppose also that $S$ is transverse to its covering translate $\rho S$. Let $T=p_{i}(S)$. Since the restriction of $p_{i}$ to the complement of Fix $(\rho)$ is an ordinary two-fold cover, we may assume that $T$ has, at worst, double arcs, coming from intersections of $S$ with $\rho S$. The simple closed curves and properly embedded arcs in $S \cap \rho S$ may contain isolated points of intersection with $\operatorname{Fix}(\rho)$, subject to the following restrictions: (i) any point in $\operatorname{Fix}(\rho)$ is in the interior of exactly one curve in $S \cap \rho S$ (since $S$ and $\rho S$ are transverse), (ii) there is at most one fixed point along any properly embedded arc in $S \cap \rho S$ (since any homeomorphism of an interval to itself with two or more fixed points is orientation preserving, while $\rho$ is locally rotation by $\pi$ around its fixed points), and (iii) any simple closed curve in $S \cap \rho S$ contains zero or two fixed points (similar to (ii)).

We will first attempt to make $S$ and $\rho S$ disjoint from $\operatorname{Fix}(\rho)$. In the one case that this is impossible, we may at least make $S$ intersect $\operatorname{Fix}(\rho)$ only in its singular point, so that in either case $T$ has at most one singular point. We will then adjust $T$ directly, in order to produce the desired embedded orbifold compressing disc.

Intersections of $S$ and $\rho S$ with Fix $(\rho)$ come in two types: simple closed curves in $S \cap \rho S$ intersecting $\operatorname{Fix}(\rho)$ in two points and properly embedded arcs in $S \cap \rho S$ intersecting $\operatorname{Fix}(\rho)$ in one point. We begin by eliminating the former. Choose a double $\operatorname{arc} \gamma$ in $T$ with two singular points $x$ and $y$ in its closure. Let $\tilde{x}$ and $\tilde{y}$ be the lifts of these points in $N_{i}$, and let $\gamma_{1}$ and $\gamma_{2}$ be the two lifts of $\gamma$ joining $\tilde{x}$ and $\tilde{y}$. Note 


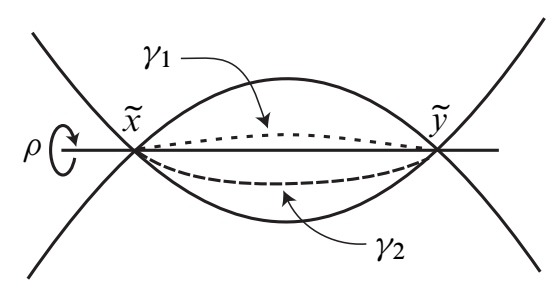

Figure 3: A simple closed curve intersecting $\operatorname{Fix}(\rho)$ twice

that $\gamma_{1} \cup \gamma_{2}$ bounds a disc $D_{S}$ in $S$ and a disc $D_{\rho S}$ in $\rho S$; see Figure 3. Assume that $D_{S}$ is innermost in $S$, and note that this implies that $D_{\rho S}$ is innermost in $\rho S$.

Now if we remove $D_{S}$ from $S$ and replace it with $D_{\rho S}$, and vice versa, and then equivariantly push off $\operatorname{Fix}(\rho)$, we get two new discs still satisfying all of the necessary hypotheses but with fewer intersections with $\operatorname{Fix}(\rho)$. Continuing this process, we may remove all remaining circles of intersection which pass through Fix $(\rho)$. For simplicity, we will refer to the resulting discs as $S$ and $\rho S$, and will continue to do so as long as it still makes sense (ie, as long as our operations are performed equivariantly on $S$ and $\rho S$ - we will later need to alter $T$ directly in ways that cannot be described via alterations in the covering space).

To finish making $S$ and $\rho S$ disjoint from $\operatorname{Fix}(\rho)$, we need just remove properly embedded arcs of intersection which pass through Fix $(\rho)$. Such an arc splits the boundary of $S$ into two pieces, which we label $a$ and $b$, coherently oriented. We will adjust $S$ and $\rho S$ in a neighborhood of the arc in such a way that each new disc will consist of one half of $S$ and one half of $\rho S$. Note that there are two ways of doing this
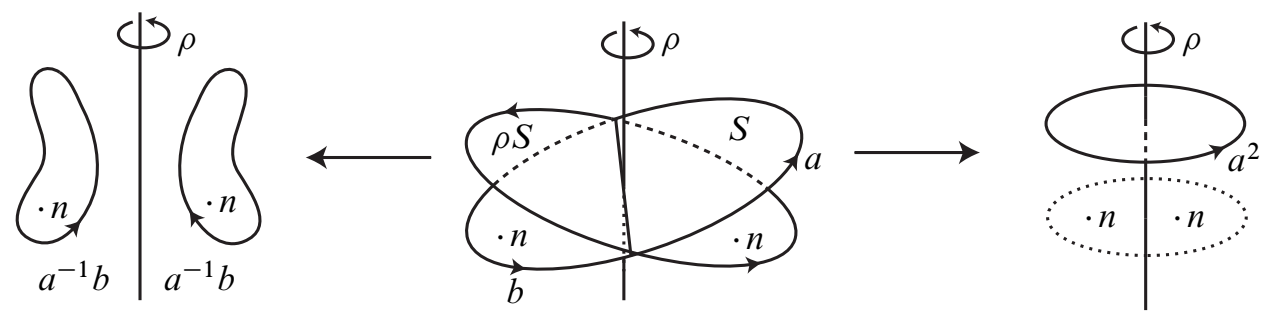

Figure 4: An embedded arc of $S \cap \rho S$ intersecting Fix $(\rho)$

(see Figure 4), both of which alter the boundary, with the new boundary equal either to $a^{-1} b$ or to $a^{2}$. We will show that one of the two resulting pairs of discs still has (the appropriate power of) its boundary mapping into $K \backslash N$.

Suppose that $S$ has singular point labeled $n$ disjoint from $\operatorname{Fix}(\rho)$, and assume it lies on the $b$ side of the arc $S \cap \rho S$. Then after cutting and pasting as described above, 
$S$ either has boundary $a^{-1} b$ and singular point labeled $n$ or has boundary $a^{2}$ and no singular point. Thus we need either $\left(a^{-1} b\right)^{n}$ or $a^{2}$ to project into $K \backslash N$. But note that

$$
(a b)^{n}=\left[a^{2}\left(a^{-1} b\right)\right]^{n-1} a^{2}\left(a^{-1} b\right)^{n}\left(a^{-1} b\right)^{-n+1} .
$$

We may think of this equation as stating that $(a b)^{n}$ is obtained from $a^{2}\left(a^{-1} b\right)^{n}$ by alternately left-multiplying by $a^{2}$ and conjugating by $a^{-1} b$, doing the pair of operations $n-1$ times. It follows that if both $\left(a^{-1} b\right)^{n}$ and $a^{2}$ map into the normal subgroup $N$, then so does $(a b)^{n}$, contrary to assumption. Thus we may remove this component of $S \cap \rho S$ by one of the two alterations discussed.

In case there is no singular point on $S$ away from $\operatorname{Fix}(\rho)$, we have, after cutting and pasting, that $S$ either has boundary $a^{-1} b$ and no singular point or has boundary $a^{2}$ and singular point labeled $n$. Thus we need either $a^{-1} b$ or $a^{2 n}$ to project into $K \backslash N$. But note that

$$
(a b)^{n}=\left(a^{-2}\right)^{n-1}\left(a^{2 n} a^{-1} b\right)\left(a^{2} a^{-1} b\right)^{n-1} .
$$

As above, we think of this as stating that $(a b)^{n}$ can be obtained from $a^{2 n}\left(a^{-1} b\right)$ by alternately conjugating by $a^{-2}$ and left-multiplying by $a^{-1} b$, doing the pair a total of $n-1$ times. It follows that one of the two described alterations produces a disc with boundary projecting into $K \backslash N$.

We may now assume that double curves in $T$ consist of simple closed curves and properly embedded arcs, all of which are disjoint from the singular set. Hereafter, we will need to alter $T$ directly, rather than indirectly via $S$.

To begin the process of removing the double curves of $T$, we first remove the simple closed curves. We do this exactly as in the manifold case, which we now sketch. For more details, see Hatcher [2]. Let $C$ be a double curve in $N_{i-1}$, and let $C_{1}$ and $C_{2}$ be the two components of $p_{i}^{-1}(C)$. A neighborhood of $C$ in $T$ is an $I$-bundle over an $X$ shape.

Assume first the bundle is trivial. Then if $C_{1}$ and $C_{2}$ cobound an annular region in $S$, we remove the image of this region from $T$, smoothing along the corner, thereby removing the double curve $C$. Otherwise, $C_{1}$ and $C_{2}$ bound distinct transverse discs, and we may exchange the images of these discs.

Now suppose the bundle is nontrivial. It must then be $X \times I$ with ends identified by a vertical (say) reflection. This is an immersed annulus, which we replace by an embedded annulus with the same boundary by splitting the top of the $X$ from the bottom. In this manner, we may remove all remaining double closed curves.

Note that we may, in this process, remove the singular point from $T$. In this case, we no longer need for $(\partial T)^{n}$ to project into $K \backslash N$, but rather for $\partial T$ to do so. But since 
the new $T$ has no singular point, it is clear that $\partial T$ maps into $K$, and the fact that some power of $\partial T$ projects into $K \backslash N$ implies that $\partial T$ does also.

We now assume that the double arcs of $T$ are all properly embedded arcs, disjoint from the image of Fix $(\rho)$. We will think of $T$ as a disc with identifications. There are two ways in which two arcs in $T$ can be identified. We will call these type (i) and type (ii) identifications, as shown in Figure 5. We require different treatments for the different ways in which a singular point may be situated on $T$ vis-à-vis the identified arcs. The case in which there is a singular point between the two arcs is similar to that in which there is no singular point at all. We therefore say $T$ is of type 1 if there is a singular point labeled $n$ between the two arcs, where $n \geq 1$; otherwise, there is a singular point elsewhere on $S$ labeled $n>1$, and we say $T$ is of type 2 . To simplify

type (i)

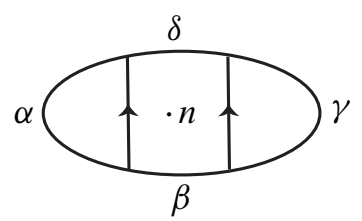

type 2

$(n>1)$

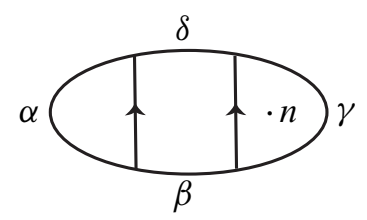

type (ii)
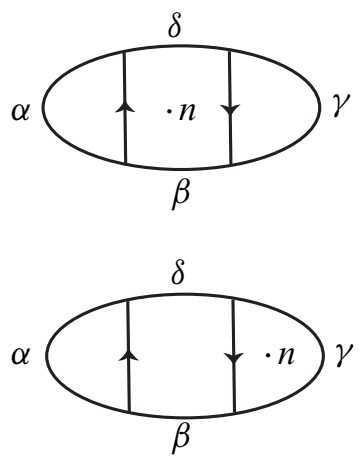

Figure 5: Four cases of identified nonsingular arcs in $T$

the discussion, we will refer to the case that $T$ is type 1 and the identification is of type (i) as Case 1(i), etc.

In all cases, we will consider the boundary of $T$ to consist of four coherently oriented $\operatorname{arcs} \alpha, \beta, \gamma, \delta$, as in Figure 5. Also, we will in each case alter $T$ in one of two ways to remove a double arc, both involving a change of the boundary. As before, it will be the case that one of the two new boundaries (or, rather, the appropriate power of such a boundary) continues to project into $K \backslash N$.

As Cases 1(i) and 1(ii) include the cases that $T$ is nonsingular, the procedure involved is identical to that used in the loop theorem for manifolds, with some minor additional verification involved to take care of the possible singular point on $T$. In Cases 1(i) and 1(ii), we cut and paste as in Figure 6. In Case 1(i), we get a disc with 

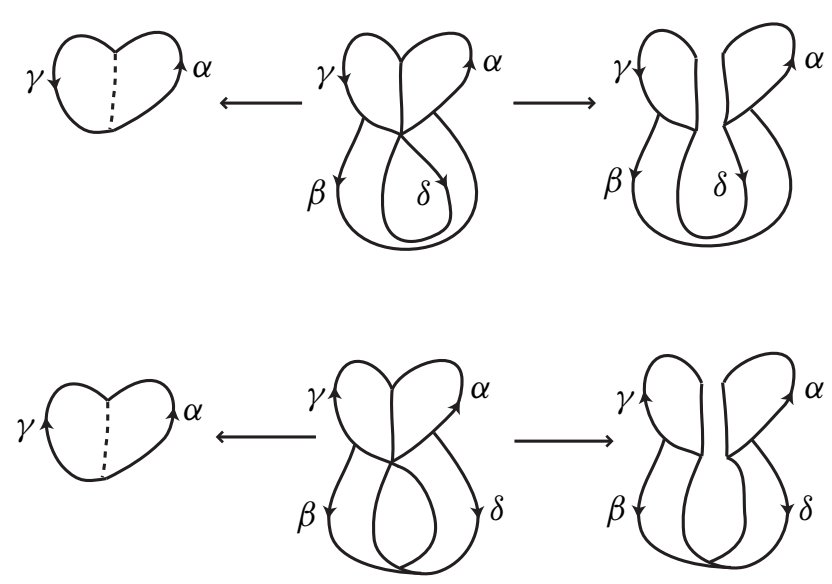

Figure 6: Case 1, the nonsingular/singular-between case

boundary either $\alpha \gamma$ or $\alpha \beta^{-1} \gamma \delta^{-1}$. In Case 1(ii), we get a disc with boundary either $\alpha \gamma^{-1}$ or $\alpha \delta \gamma \beta$. We then observe that both

$$
\delta^{-1}\left[\left(\alpha \beta^{-1} \alpha^{-1}\right)(\alpha \gamma) \delta^{-1}\right]^{n}\left(\alpha \beta^{-1} \gamma \delta^{-1}\right)^{-n}\left[\delta\left(\alpha \beta \alpha^{-1}\right)(\alpha \gamma)\right]^{n} \delta
$$

and $\alpha \beta\left[\left(\beta^{-1} \alpha^{-1}\right)\left(\alpha \gamma^{-1}\right)\left(\delta^{-1} \gamma^{-1}\right)\left(\alpha \gamma^{-1}\right)^{-1}\right]^{n}(\alpha \delta \gamma \beta)^{n}[(\gamma \delta)(\alpha \beta)]^{n} \beta^{-1} \alpha^{-1}$

are equal to $(\alpha \beta \gamma \delta)^{n}$, for $n \geq 1$. We read the first equation as raising $\alpha \beta^{-1} \gamma \delta^{-1}$ to the $-n$; conjugating by $\delta^{-1}$, left-multiplying by $\alpha \gamma$, conjugating by $\alpha \beta^{-1} \alpha^{-1}$, and right-multiplying by $\alpha \gamma$, a total of $n$ times; and then finishing off by conjugating by $\delta^{-1}$. We read the second equation as raising $\alpha \delta \gamma \beta$ to the $n$; left-multiplying by $\left(\alpha \gamma^{-1}\right)^{-1}$, conjugating by $\delta^{-1} \gamma^{-1}$, left-multiplying by $\alpha \gamma^{-1}$, and conjugating by $\beta^{-1} \alpha^{-1}$, a total of $n$ times; and then finishing off by conjugating by $\alpha \beta$. So in either case, there is a product of conjugates of the two possible boundaries projecting outside of the normal subgroup $N$. It therefore cannot be that both possible boundaries project into $N$. We now replace $T$ with the altered disc having boundary projecting into $K \backslash N$, and note that this new disc has (at least) one less double arc than did $T$.

For Cases 2(i) and 2(ii), we assume that the identified $\operatorname{arc}$ in $T$ further from the singular point is outermost among all identified arcs. In these cases, we consider a different option for altering $T$. We will assume the singular point lies on the $\gamma$ side of the identified arcs in $T$. In each case, for one of the two possible new discs, we still consider that with boundary $\alpha \gamma$ (in Case 2(i)) or $\alpha \gamma^{-1}$ (in Case 2(ii)). For the other option, we consider a disc with boundary isotopic $\alpha \beta \alpha^{-1} \delta$ (in Case 2(i)) or $\alpha \delta \alpha \beta$ (in Case 2(ii)), making use of a parallel copy of the projection of the (nonsingular) piece of $T$ with $\alpha$ in its boundary; see Figure 7 . 

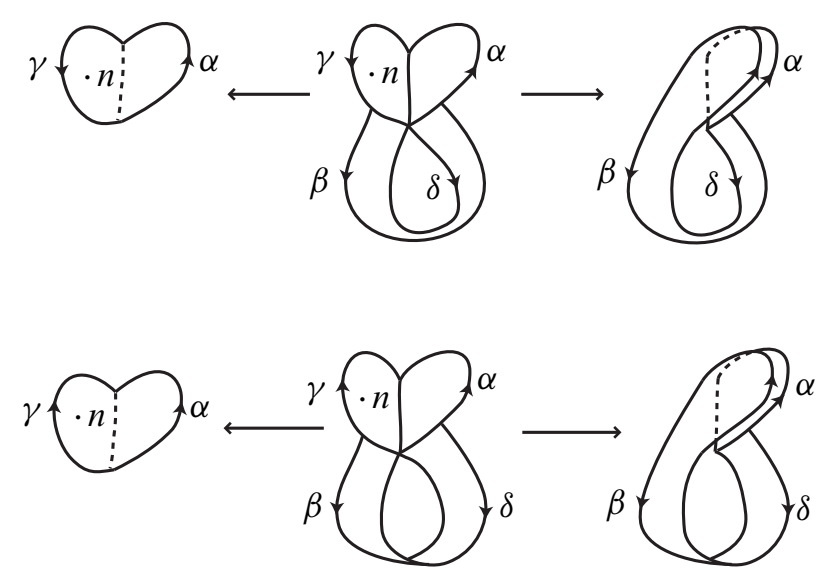

Figure 7: Case 2, when there is a singular point not between the identified arcs

We now consider the following expressions:

$$
\left(\alpha \beta \alpha^{-1} \delta\right)\left[(\alpha \beta \gamma \delta)^{-1}\left(\alpha \beta \alpha^{-1} \delta\right)\right]^{n-1} \delta^{-1}(\alpha \gamma)^{n} \delta[\alpha \beta \gamma \delta]^{n-1},
$$

which is formed by raising $\alpha \gamma$ to the $n$; conjugating by $\delta^{-1}$; left-multiplying by $\alpha \beta \alpha^{-1} \delta$ and then conjugating by $\alpha \beta \gamma \delta$, a total of $n-1$ times; and finally leftmultiplying by $\alpha \beta \alpha^{-1} \delta$, and

$$
(\gamma \delta)^{-1}\left[\left(\alpha \gamma^{-1}\right)^{-1}(\gamma \delta \alpha \beta)^{-1}\right]^{n-1}\left(\alpha \gamma^{-1}\right)^{-1}(\alpha \delta \alpha \beta)^{n}[\gamma \delta \alpha \beta]^{n-1}(\gamma \delta),
$$

which is formed by raising $\alpha \delta \alpha \beta$ to the $n$; left-multiplying by $\left(\alpha \gamma^{-1}\right)^{-1}$; conjugating by $(\gamma \delta \alpha \beta)^{-1}$ and then left-multiplying by $\left(\alpha \gamma^{-1}\right)^{-1}$, a total of $n-1$ times; and finally conjugating by $(\gamma \delta)^{-1}$.

Both of these expressions are equal to $(\alpha \beta \gamma \delta)^{n}$, which implies, as above, that in both Case 2(i) and Case 2(ii), there is a choice of alterations of $T$ that results in a disc having (at least) one less double arc and whose boundary projects into $K \backslash N$.

Repeating this process, we remove all remaining double arcs, thereby producing an embedded orbifold disc in $N_{i-1}$ whose boundary maps into $K \backslash N$, moving us one level down the tower. Continuing to the case $i=1$ completes the proof.

As in the manifold case, we have the following corollary.

Corollary Let $Q$ be a 3-orbifold for which the conclusion of the loop theorem holds (eg, $Q$ is good, or has singular set labeled with powers of 2). Let $F$ be a two-sided orbifold incompressible 2-suborbifold of $Q$, and let $p: \hat{Q} \rightarrow Q$ be a finite orbifold cover. Then $p^{-1}(F)$ is orbifold incompressible in $\widehat{Q}$. 
Proof Suppose $p^{-1}(F)$ is orbifold compressible, and let $f: D \rightarrow \widehat{Q}$ be an orbifold compressing disc with $f(\partial D)=\gamma \subset p^{-1}(F)$. We may assume that all circles in $f(D) \cap p^{-1}(F)$ are trivial in $\pi_{1}^{\text {orb }}\left(p^{-1}(F)\right)$, by passing to innermost (in $D$ ) essential (in $\left.\pi_{1}^{\text {orb }}\left(p^{-1}(F)\right)\right)$ circles. Then each circle in $f(D) \cap p^{-1}(F)$ bounds a disc in $p^{-1}(F)$. Cut and paste along each of these circles (beginning with those innermost in $D$ ), removing the intersections by replacing each portion of $f(D)$ bounded by such a circle with a parallel copy of the disc in $p^{-1}(F)$ the circle bounds. Since the boundary has not changed, we now have an orbifold compressing disc $f: D \rightarrow \widehat{Q}$ with $f(D) \cap p^{-1}(F)=f(\partial D)$. Composing with $p$, we obtain a wound disc $(p \circ f): D \rightarrow Q$ with $(p \circ f)(D) \cap F=(p \circ f)(\partial D)$. Splitting $Q$ open along $F$, we obtain an orbifold $Q^{\prime}$ containing a wound disc $g: D \rightarrow Q^{\prime}$ with $g(\partial D) \subset F$. The loop theorem then provides an orbifold compressing disc with boundary in $F$, and the result follows by gluing $Q$ back together.

Acknowledgments I gratefully acknowledge the support and encouragement of the Mathematics Department at the University of California, Santa Barbara, where this paper was written while the author was a graduate student. In particular, I thank Darren Long, who taught me the loop theorem, and Daryl Cooper, who told me about orbifolds. Many thanks to the referee for a careful reading and helpful comments.

\section{References}

[1] D Cooper, C D Hodgson, S P Kerckhoff, Three-dimensional orbifolds and conemanifolds, MSJ Memoirs 5, Math. Soc. of Japan, Tokyo (2000) MR1778789 With a postface by S Kojima

[2] A Hatcher, Notes on basic 3-manifold topology, book draft (2007) Available at http://www . math. cornell. edu/ hatcher/3M/3Mdownloads.html

[3] CD Papakyriakopoulos, On Dehn's lemma and the asphericity of knots, Ann. of Math. (2) 66 (1957) 1-26 MR0090053

[4] Y Takeuchi, M Yokoyama, PL-least area 2-orbifolds and its applications to 3orbifolds, Kyushu J. Math. 55 (2001) 19-61 MR1825821

Department of Mathematics \& Statistics, University of South Alabama ILB 325, 307 North University Blvd, Mobile AL 36688, USA

jbarnard@jaguar1.usouthal.edu

http://www. southalabama.edu/mathstat/personal_pages/jbarnard/

Received: 18 May 2011 Revised: 23 August 2011 